\title{
Improved Bend Loss Formula Verified for Optical Fiber by Simulation and Experiment
}

\author{
Ross T. Schermer, Member, IEEE, and James H. Cole
}

\begin{abstract}
This paper presents an improved curvature loss formula for optical waveguides, which is shown to accurately predict the bend loss of both single-mode and multimode fibers. The formula expands upon a previous formula derived by Marcuse, greatly improving its accuracy for the case of multimode fiber. Also presented are the results of bent fiber simulations using the beam propagation method (BPM), and experimental measurements of bend loss. Agreement among simulation, formula and measurement support the validity of both theoretical methods. BPM simulations showed that the lowest order modes of the bent fiber were reduced to their linearly polarized constituents prior to the onset of significant bend loss. This implies that certain LP mode orientations should propagate with much lower loss than previously expected, and should impact the mode stripping ability of bent large mode area fibers, as employed in fiber lasers and amplifiers.
\end{abstract}

Index Terms-Dielectric waveguides, laser amplifiers, optical fiber amplifiers, optical fiber lasers, optical waveguide theory, waveguide bends.

\section{INTRODUCTION}

$\mathbf{V}$ ARIOUS theoretical methods exist to predict curvature loss in optical waveguides. The usual approach is to use a simplified formula introduced by Marcuse [1], applicable to weakly guided waveguides, including most optical fibers, for sufficiently large radii of curvature. This formula agrees well with experiment for single-mode fiber, after adjustments are made for bend-induced stress [2]. However, for multimode fiber it can be quite inaccurate [3]. Other analytical bend loss formulae are similarly limited, in that none are known to reliably predict mode-dependent bend loss in multimode fibers. This has become a significant problem with the development of the coiled multimode fiber amplifier [4], which uses bend loss to strip out the higher order fiber modes, and thereby achieve single-mode, large mode area operation. Optimizing these devices, and understanding their ultimate limitations, requires that mode-dependent bend loss be predicted accurately.

An alternative to analytical formulae for predicting bend loss is to use numerical simulation. Various methods have been developed to simulate field propagation in dielectric waveguides [5]. The beam propagation method (BPM) [5], in combination with conformal mapping [6], is well-suited to curved waveguides because it allows one to rapidly determine the modes of

Manuscript received January 22, 2007; revised April 19, 2007. This work was supported by a Postdoctoral Research Associateship from the National Research Council.

The authors are with the Optical Sciences Division of the U.S. Naval Research Laboratory, Washington, DC 20375 USA (e-mail: schermer@ccs.nrl.navy.mil; jim.cole@nrl.navy.mil).

Color versions of some or all figures are available online at http://ieeexplore. ieee.org.

Digital Object Identifier 10.1109/JQE.2007.903364 a structure, and then propagate them over large distances, while using relatively little computer memory. Both tasks may be done with the same software package. However, although bend loss has been studied with BPM in the past [7], [8], the accuracy of this method has yet to be firmly established.

This paper studies bend loss in step-index, single-mode and multimode fibers using BPM and conformal mapping, and compares these results to both analytical models and experiment. One aim is to establish the accuracy of the BPM simulations at predicting bend loss, for both the fundamental and higher order modes of a simple waveguide. Another is to clarify the sources of error in Marcuse's simplified bend loss formula, and seek to improve its accuracy for multimode fiber. The final goal is to demonstrate an accurate, quasi-analytical formula for bend loss, which is reliable provided that the mode field distribution of the bent waveguide is known.

Section II begins by describing the simulation method used in this paper, as well as the modes calculated for the bent fiber and typical propagation results. Section III compares the simulated bend loss to analytical predictions and experimental results. The simulations are shown to accurately predict experimentally measured fundamental mode bend loss, for both single-mode and multimode fibers. By comparison, the simplified loss formula is only in agreement for the single-mode case. Section IV analyzes this discrepancy in detail, and finds that by removing an unnecessary term in the loss formula, its accuracy is greatly improved. The BPM-simulated bend loss is then shown to be in excellent agreement with a quasi-analytical formula, modified from Marcuse's original derivation. This latter result holds for both the fundamental and higher order modes. This knowledge is used in Section V to gauge the dominant sources of error in the simplified bend loss formula, and is followed by a discussion and summary of results.

\section{BENT Fiber Simulation}

Bent fiber simulations were performed in this paper using a finite difference approach, the beam propagation method (BPM), in conjunction with the conformal mapping technique. The first step in the process was to transform the circularly curved fiber to an equivalent, straight fiber by the process of conformal mapping [6], [9]. This process is shown schematically in Fig. 1. Coordinate transformation allowed the bent fiber to be represented by an equivalent, straight fiber, with modified refractive index distribution, $n^{\prime}(x, y)$

$$
n^{\prime}=n_{\text {material }} \exp \left(\frac{x}{R}\right) \approx n_{\text {material }}\left(1+\frac{x}{R}\right) .
$$

Here $n_{\text {material }}(x, y)$ is the refractive index of the bent waveguide cross-section, and the exponential term accounts for the increase 


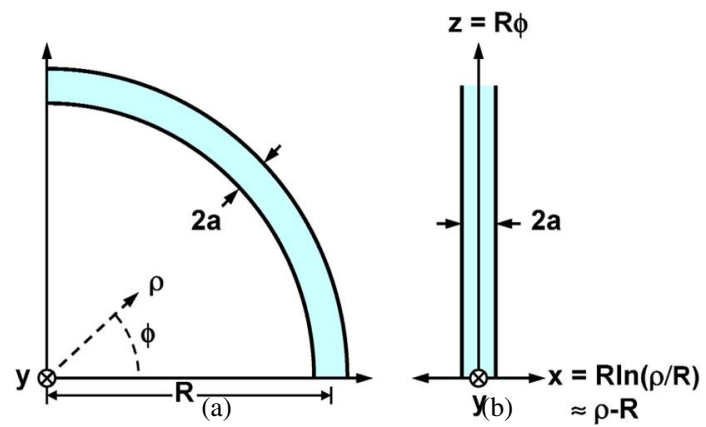

Fig. 1. (a) Schematic diagram of a circularly bent fiber and (b) the equivalent, straight fiber after conformal mapping. Light is guided along the $\phi$ and $z$ directions, respectively.

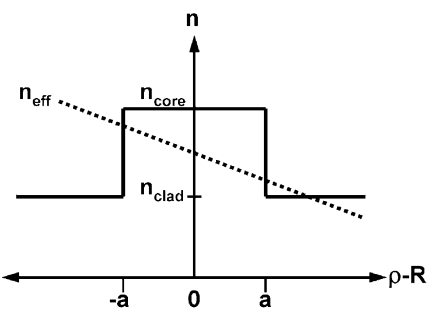

(a)

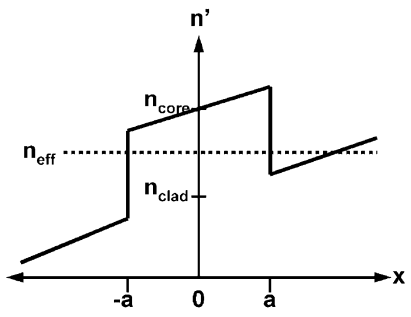

(b)
Fig. 2. (a) Refractive index distribution of an unstressed, bent fiber and (b) that of its equivalent, straight fiber after conformal mapping. The slice shown is through the center of the fiber. The refractive index of the conformal mapped fiber increases away from the center of curvature. Variation in the modal effective index, $n_{\text {eff }}$, is also shown in each case.

in optical path length along the fiber with distance from the center of curvature. Relatively slow bends $(x \ll R)$ were assumed in this paper to allow the first-order approximation used in (1). The resulting index distribution was tilted with respect to the original, increasing away from the center of the bend, as shown in Fig. 2.

An additional change occurs to the physical refractive index of the fiber upon bending, due to stress-optic effects. Compression along the inner half of the fiber, towards the center of the bend, and tension along the outer half, cause the material refractive index to vary according to the relation [10], [11]

$$
n_{\text {material }}=n\left[1-\frac{n^{2} x}{2 R}\left[P_{12}-\nu\left(P_{11}+P_{12}\right)\right]\right] \text {. }
$$

Here $n(x, y)$ is the refractive index of the straight fiber, $\nu$ is Poisson's ratio, and $P_{11}$ and $P_{12}$ are components of the photoelastic (or elasto-optical) tensor. Again, the refractive index tilts with bending, but in this case it typically decreases toward the outside of the bend.

Combining (1) and (2), defining the equivalent bend radius as

$$
R_{\mathrm{eff}} \equiv \frac{R}{1-\frac{n^{2}}{2}\left[P_{12}-\nu\left(P_{11}+P_{12}\right)\right]}
$$

and simplifying to first order then leads to an expression similar to (1), but in terms of the unperturbed index $n(x, y)$

$$
n^{\prime}=n\left(1+\frac{x}{R_{\mathrm{eff}}}\right) .
$$

This is the refractive index distribution pertaining to the equivalent, straight waveguide that was used for the BPM simulations

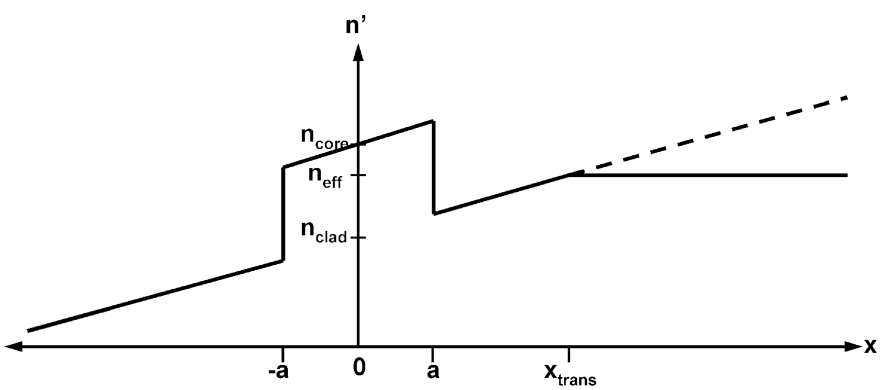

Fig. 3. Conformal mapped index distribution used for BPM mode calculations (solid line). The sloping index distribution was truncated at the point $x=x_{\text {trans }}$ in order to prevent significant loss to the cladding during the mode calculations. By setting $x_{\text {trans }}$ just within the caustic boundary, error due to this perturbation was minimized.

in this paper. The bracketed term represents the net effects of bending, and the effective bend radius accounts for the dominant stress effects. Note that for silica fiber, $R_{\text {eff }} / R \approx 1.28$ [2], so stress actually counteracts the effects of bending compared to curvature alone [12].

Given the equivalent, straight fiber represented by (4), it was not difficult to determine the modes of the structure, and then simulate their propagation, using standard BPM techniques. Since the beam propagation method has been well-documented elsewhere [5], it should suffice to state that the approach solves Maxwell's equations numerically, by representing the fields and refractive index distribution on a uniform transverse $(\Delta x, \Delta y)$ grid, and stepping in equal steps $(\Delta z)$ along the waveguide to simulate wave propagation. Of the various BPM versions available, a semi-vectorial variation was used in this paper, as described in [13]. At the simulation boundaries, transparent boundary conditions were used to minimize unwanted reflections [14].

The semi-vector BPM was chosen over a full-vector alternative because it proved to be faster, more stable, and less sensitive to step size. An apparent disadvantage was that it did not account for polarization coupling within the fiber. This limited analysis to the linearly polarized (LP) modes, rather than the more precise hybrid (HE and $\mathrm{EH}$ ) fiber modes [15]. However, as will be seen, this limitation proved to be inconsequential when the fiber was bent to the point of significant loss.

The lowest order LP modes of the bent fiber were calculated using the imaginary-distance BPM technique [5], with the refractive index distribution given by (4). This was done for a variety of core radii $(a)$, effective bend radii $\left(R_{\text {eff }}\right)$, unperturbed core and cladding indices ( $n_{\text {core }}$ and $\left.n_{\text {clad }}\right)$, and wavelengths $\left(\lambda_{o}\right)$. In order to prevent loss to the cladding during the mode calculations, which was inevitable for tight bends, and which hindered convergence, the cladding refractive index was truncated for larger values of $x$ as shown in Fig. 3. Although this perturbation represented a source of error in the mode calculations, the error was minimized by moving the transition point, $x_{\text {trans }}$, as far out as possible without introducing significant loss. This meant setting $x_{\text {trans }}$ just within the caustic boundary, given by [16]

$$
x_{\text {caustic }}=R_{\text {eff }}\left(\frac{n_{\text {eff }}}{n_{\text {clad }}}-1\right)
$$


where $n_{\text {eff }}=\operatorname{real}\left(\beta_{z}\right) / k_{o}, \beta_{z}$ is the modal propagation constant, and $k_{o}=2 \pi / \lambda_{o}$. At the caustic boundary the mode speed matches the speed of light in the cladding, and beyond it the fields become radiative. Approximate mode solutions were first calculated by setting $x_{\text {trans }}=a$ in the simulations, and the resulting propagation constant was used to estimate $x_{\text {caustic }}$. Then the simulations were repeated with $x_{\text {trans }}$ just within the caustic boundary, in order to obtain the modes of the bent fiber. Step sizes used in the simulations were typically $\Delta x=\Delta y=$ $0.1 \mu \mathrm{m}$ and $\Delta z=0.5 \mu \mathrm{m}$. For core radii greater than $25 \mu \mathrm{m}$, however, the transverse step was increased to $\Delta x=\Delta y=$ $0.2 \mu \mathrm{m}$ to allow a larger simulation area. In each case, increasing or decreasing the step sizes had negligible impact on the propagation constants and simulated loss, so they appeared to be adequate.

Fig. 4 displays typical results of mode calculations for the lowest order fiber modes. Conditions for this example were $a=$ $12.5 \mu \mathrm{m}, \lambda_{o}=1.064 \mu \mathrm{m}, \mathrm{NA}_{\text {index }}=0.1$, where

$$
\mathrm{NA}_{\text {index }}=\sqrt{n_{\text {core }}^{2}-n_{\text {clad }}^{2}}
$$

and $n_{\text {clad }}=1.52$, which corresponded to a particular fiber of interest. For the case of the bent fiber, the effective bend radius of $1.24 \mathrm{~cm}$ was chosen to display the modes when the fundamental mode was relatively stable, while the others exhibited significant loss. As expected from previous work [3], bending tended to distort the fiber modes, and caused them to shift away from the center of curvature. Furthermore, the two orientations of each $\mathrm{LP}$ mode, denoted here as $\mathrm{LP}_{\mathrm{mne}}$ and $\mathrm{LP}_{\mathrm{mno}}$ for whether they were even or odd along the $y$ direction, became noticeably different upon bending. Whereas in the straight fiber they were identical other than a $\pi / 2$ rotation, bending destroyed this rotational symmetry.

Such profound changes in the fiber mode distributions also manifested themselves in significant changes in the modal propagation constants compared to the straight fiber. A useful measure of this effect was the normalized propagation constant, defined for the bent fiber as

$$
b_{\text {bent }}=\left.\frac{n_{\text {eff }}^{2}-n_{\text {clad }}^{\prime 2}}{n_{\text {core }}^{\prime 2}-n_{\text {clad }}^{\prime 2}}\right|_{x=a} .
$$

So defined, all the guided modes of the bent fiber must satisfy the relation $0<b_{\text {bent }}<1$. Fig. 5 plots $b_{\text {bent }}$ versus $R_{\text {eff }}$ for the modes in Fig. 4. As shown, the propagation constants of the $\mathrm{LP}_{11 \mathrm{e}}$ and $\mathrm{LP}_{11 \text { o }}$ modes deviated substantially from each other when the fiber was adequately bent. The same was true of the $\mathrm{LP}_{21 \mathrm{e}}$ and $\mathrm{LP}_{21 o}$ modes, although to a lesser extent. Fig. 5 also notes the effective bend radius where each mode began to radiate appreciably $(0.1 \mathrm{~dB} / \mathrm{m})$. This shows that by the time the even-odd mode pairs reached the point of significant loss, they had significantly different propagation constants, and were therefore no longer velocity-matched.

An important implication of this is that without precise velocity matching, the even-odd mode pairs of the fiber should not be expected to maintain significant polarization coupling with one another. The $\mathrm{TE}_{01}, \mathrm{TM}_{01}$, and $\mathrm{HE}_{21}$ modes of the straight
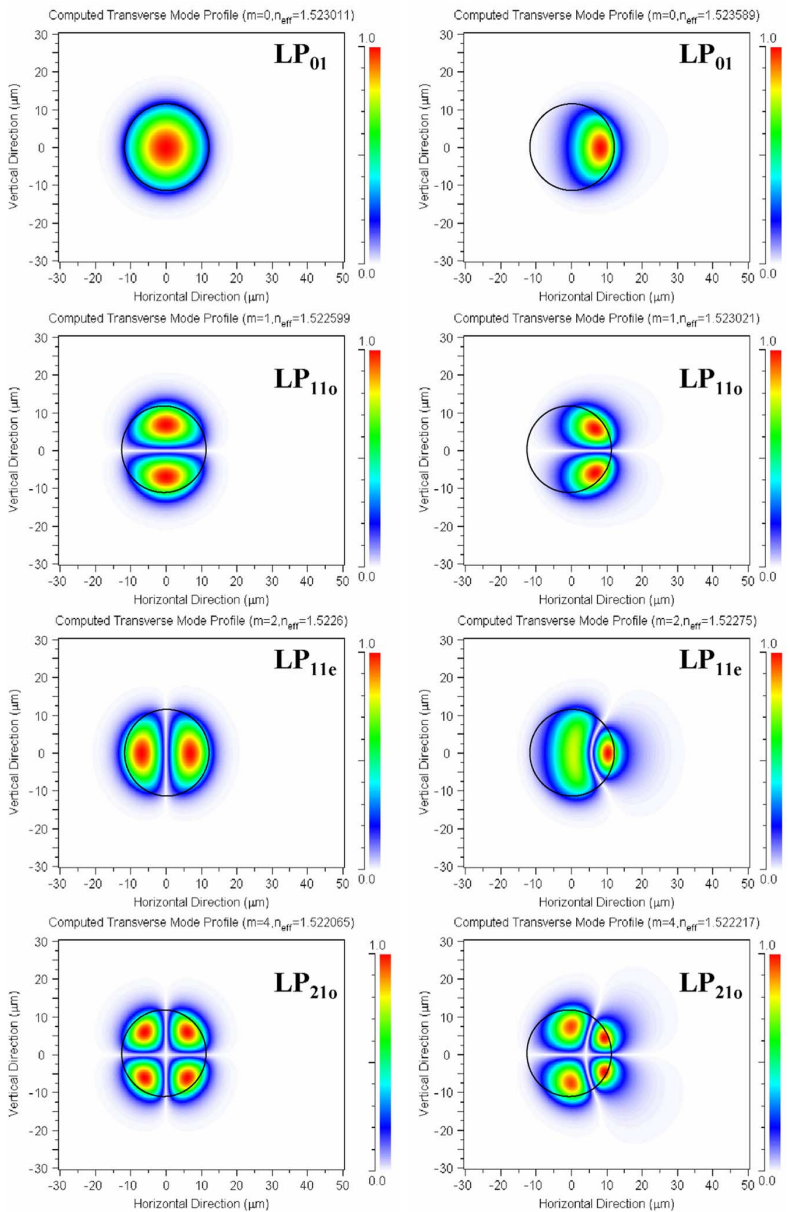

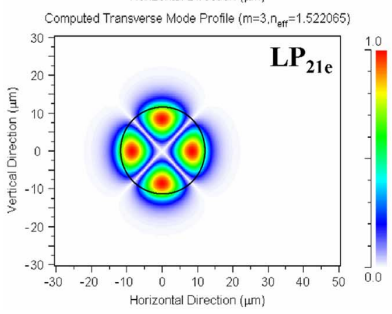

(a)

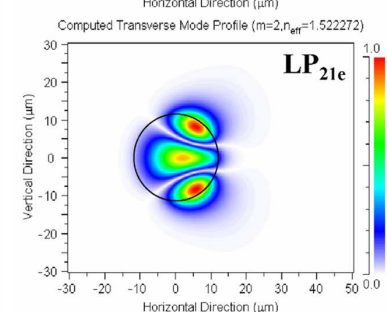

(b)
Fig. 4. Simulated mode field distributions of the lowest order fiber modes, for (a) straight fiber and (b) bent fiber. Electric field magnitude is displayed on the scale to the right, and the core-cladding interface is denoted by the circular outline. For bent fiber modes, the center of curvature is located to the left of each figure. The subscripts " $e$ " or " $o$ " added to the mode labels denote whether the mode is even or odd with respect to the $x$ axis, respectively. In the straight fiber the $\mathrm{LP}_{\mathrm{mne}}$ and $\mathrm{LP}_{\mathrm{mno}}$ modes of opposite polarization are coupled. In the bent fiber they are no longer degenerate, which can prevent significant polarization coupling.

fiber, which are comprised of different combinations of polar-

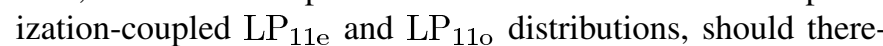
fore be expected to transition into their LP constituents given adequate bending. The same also holds for higher order modes (although at discrete values of $R_{\text {eff }}$, velocity matching may be possible, as may be inferred from Fig. 9). Simulations also showed that as fiber $V$ number was increased, where $V$ is defined

$$
V=k_{o} a \sqrt{n_{\text {core }}^{2}-n_{\text {clad }}^{2}}
$$

the LP mode degeneracy was more readily broken. 


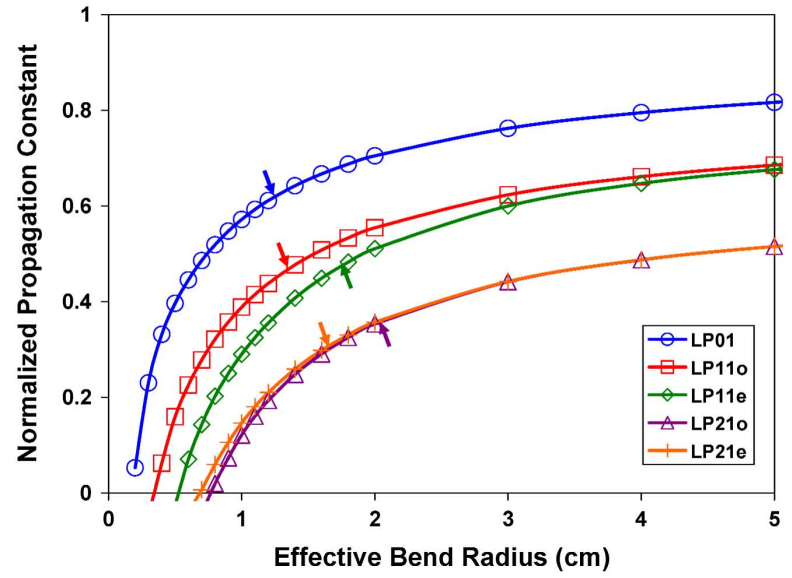

Fig. 5. Simulated normalized propagation constants $b_{\text {bent }}$ of the lowest order fiber modes of a bent fiber. For large bend radii, the propagation constants of the $\mathrm{LP}_{\text {mne }}$ and $\mathrm{LP}_{\text {mno }}$ modes are similar. However, with adequate bending they can differ substantially. The deviation is less pronounced for higher order modes. The arrows mark the bend radii where simulated bend loss was $0.1 \mathrm{~dB} / \mathrm{m}$. The propagation constants of the even and odd modes differed substantially before the onset of significant bend loss.

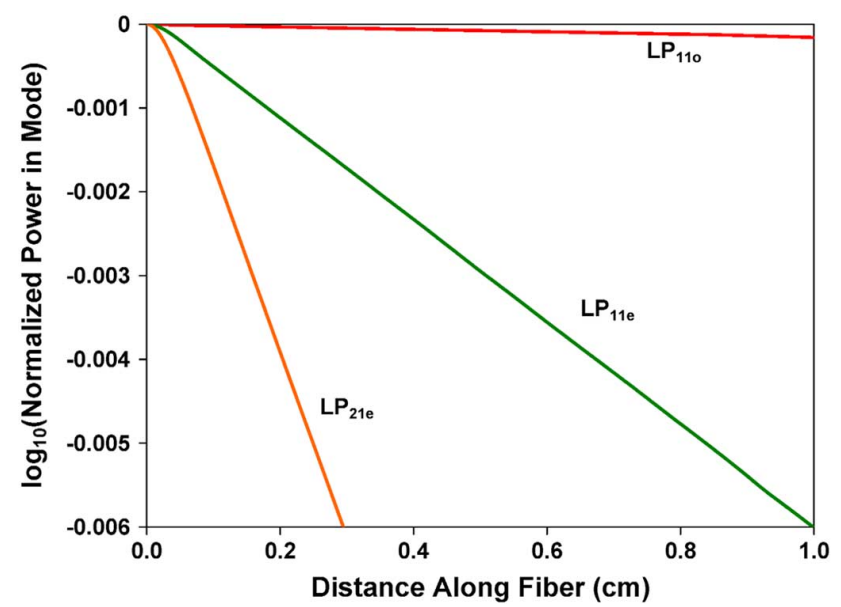

Fig. 6. Mode power versus distance from various BPM simulations. For each simulation, the mode field distribution was launched and propagated with the BPM. Power in the launched mode was monitored using the overlap integral between the propagating fields and the mode field distribution. Bend loss was inferred from the slope of each curve.

Having shown that the relevant modes of the fiber were the LP modes as far as bend loss was concerned, the next step was to simulate their propagation loss. This was accomplished by launching a given mode field distribution into the fiber (the initial condition), and then monitoring its power as the fields were propagated with the BPM. Power was monitored by calculating the overlap integral of the fields in the simulation region with those of the mode field distribution, at uniform steps along the fiber. Results of a typical loss measurement are shown in Fig. 6. Pure exponential power decay was typical of higher order modes as well as the fundamental mode. This was possible because the modes were well-orthogonalized by the mode solver, with typical inter-mode overlaps less than $-30 \mathrm{~dB}$. As a result, measured decay corresponded to that of an individual mode rather than their combination.
The propagation simulations were performed using the same transverse and longitudinal step sizes as the mode calculations. Simulation regions were $120 \times 60 \mu \mathrm{m}^{2}$ for core radii $25 \mu \mathrm{m}$ or less, and $240 \times 120 \mu \mathrm{m}^{2}$ for larger cores. This allowed the modes to be propagated about $1 \mathrm{~cm}$ along the fiber before reflections from the boundaries began to impact the measurements. Given the high accuracy of the overlap calculation, bend loss could be simulated with this technique down to about $0.01 \mathrm{~dB} / \mathrm{m}$.

Simulations performed for different polarizations showed that there was no clear polarization dependence to the loss, in agreement with theoretical predictions for weakly guided fiber [17]. Selected simulations were also repeated with $n^{\prime}$ expanded to second order in $x / R$. These did not exhibit substantial variations in losses or propagation constants compared to the first-order approximation in (1).

\section{COMPARISON WITH EXPERIMENT}

In order to test the accuracy of both the BPM simulations in this paper and the simplified bend loss formula presented by Marcuse, both were compared to experimental bend loss data. The simplified bend loss formula for optical fiber [1], modified here to include fiber stress through use of the effective bend radius, is given by

$$
2 \alpha=\frac{\pi^{1 / 2} \kappa^{2} \exp \left(-\frac{2 \gamma^{3} R_{\mathrm{eff}}}{3 \beta_{z}^{2}}\right)}{2 R_{\mathrm{eff}}^{1 / 2} \gamma^{3 / 2} V^{2} K_{m-1}(\gamma a) K_{m+1}(\gamma a)} .
$$

Here $2 \alpha$ is the power loss coefficient, $\kappa$ and $\gamma$ are the field decay rates in the core and cladding

$$
\begin{aligned}
& \kappa=\sqrt{k_{\text {core }}^{2}-\beta_{z}^{2}} \\
& \gamma=\sqrt{\beta_{z}^{2}-k_{\text {clad }}^{2}}
\end{aligned}
$$

the $K$ terms are modified Bessel functions, and $m$ is azimuthal mode number (equal half the number of azimuthal zeros), corresponding to the subscript in $\mathrm{LP}_{\mathrm{mn}}$. Note that this formula is only applicable to even LP modes. Bend loss in units of $\mathrm{dB} /$ length is obtained by multiplying $2 \alpha$ by the factor 4.343 .

The first fiber tested was Corning SMF-28, a step-index, single mode fiber. Two sets of experimentally measured bend loss data [18] are shown in Fig. 7, for the wavelengths 1320 and $1550 \mathrm{~nm}$. Also shown are bend loss curves calculated by BPM simulation, and by (9). As can be seen, both theoretical methods agree well with each other, as well as with the measured data. Fiber parameters used for the theoretical predictions were $2 a=8.2 \mu \mathrm{m}$, and $n_{\text {clad }}=1.447$ and 1.440 at $\lambda_{o}=1.32 \mu \mathrm{m}$ and $1.55 \mu \mathrm{m}$, respectively. The quantity $\mathrm{NA}_{\text {index }}$ was estimated from specifications for fiber cutoff wavelength and mode field diameters to be 0.117 , as discussed in Appendix B. The propagation constants used in (9)-(11) were determined using standard numerical techniques for straight fiber [19], and were in good accord with simulated values for straight SMF-28 fiber. Note that it was necessary to account for stress in the predictions, through use of the effective bend radius, to obtain such agreement between theory and experiment. 


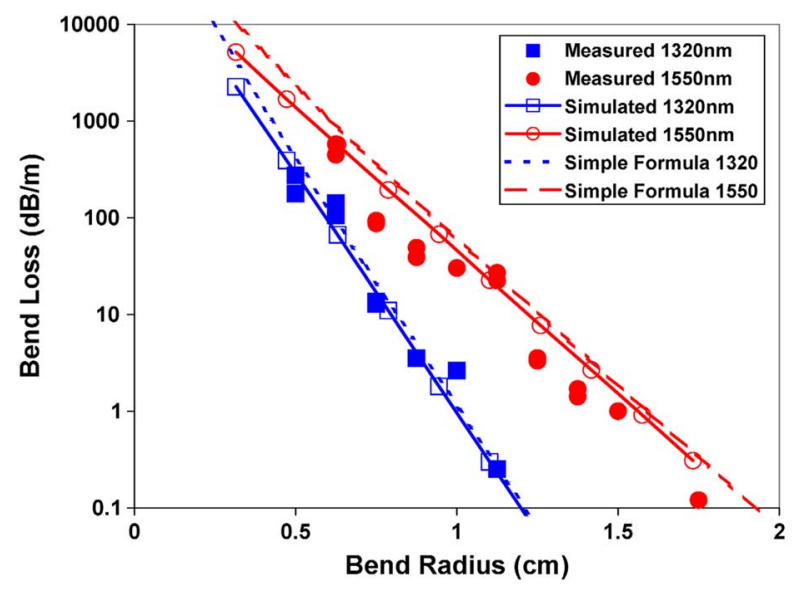

Fig. 7. Comparison of bend loss data for SMF-28 fiber, from measurement, BPM simulation, and simplified loss formula, (9). Simulated data points are as marked. For this single mode fiber $(V=2.28$ at $1320 \mathrm{~nm}, V=1.94$ at 1550 $\mathrm{nm})$, the simulated and formulaic bend loss was quite similar. Both agreed with experiment at the wavelength $1320 \mathrm{~nm}$, and reasonably well at $1550 \mathrm{~nm}$. Stress was accounted for in both simulation and formula, which was necessary for the good agreement.

Measurements were also performed on a multimode fiber from Liekki, model Passive 25/240DC. This fiber had a 25- $\mu$ m core diameter, cladding index of 1.46 , and $\mathrm{NA}_{\text {index }}$ of 0.06 , yielding $V$ numbers of 7.44 and 5.67 at the test wavelengths of 633 and $830 \mathrm{~nm}$, respectively. Due its multimode nature $(V>2.405)$, loss measurements were restricted to the fundamental mode, which experiences the least amount of loss when the fiber is bent. This allowed the higher order modes to be stripped out, leaving predominantly the fundamental mode in the core at the output. To test the bend loss, the fiber was wound on rods of various sizes, and its transmission measured with a laser and photodetector. Since light guided by the cladding was a source of error in the measurements, the low-index fiber jacket was removed before winding the fiber, and replaced by a coating of black ink. This adequately extinguished the light in the cladding before it reached the spool, and removed the light lost to the cladding while traversing the bend. The photo-detector was mounted directly on the rod so that transmission measurements could be taken while winding the fiber. This minimized variations in the transition loss between the bent and straight fiber regions. Error in the measurement due to higher order modes and transition loss was also minimized by neglecting the initial portion of the decay, and fitting only the pure exponential. Typically, the length of fiber was adjusted to provide $20 \mathrm{~dB}$ of pure fundamental mode loss for each bend radius measured. Results of the bend loss measurements are shown in Fig. 8, and exhibit the usual exponential dependence on bend radius.

Also shown in Fig. 8 are bend loss curves from BPM simulations and the simplified bend loss formula, (9). As indicated in the figure, and discussed previously in [3], (9) vastly overestimates the fundamental mode bend loss in multimode fiber. In contrast, the results of the BPM simulations were in good agreement with the measured data.

Such agreement between BPM simulations and measurement, for both single-mode and multimode fiber, strongly suggests the accuracy of the BPM approach. This is interesting because the simulations were not based on any assumptions

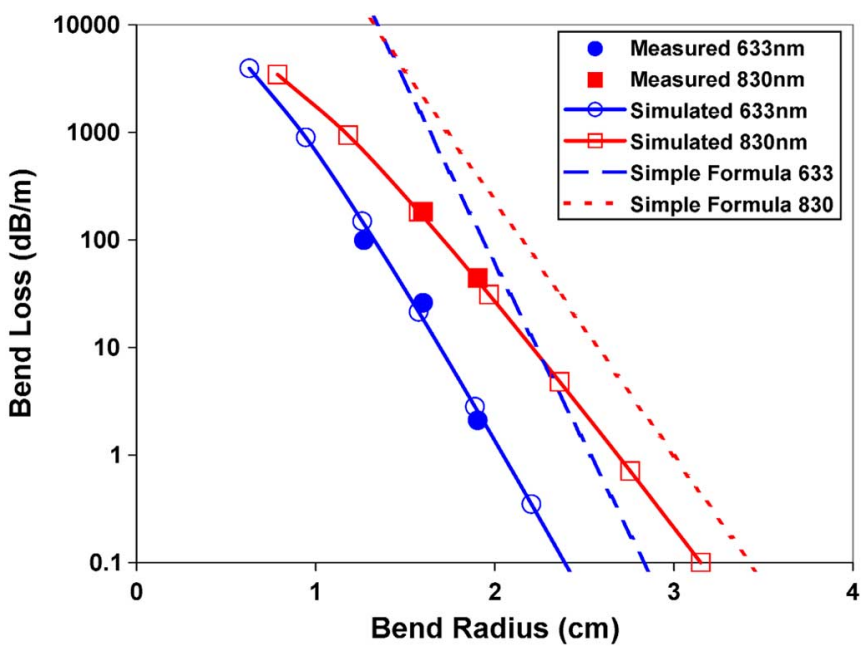

Fig. 8. Comparison of fundamental mode bend loss data for Liekki passive 25/240DC fiber, from measurement, BPM simulation, and simplified loss formula, (9). Simulated data points are as marked. The simulated data was in good agreement with experiment at both wavelengths. However, for this multimode fiber $(V=7.44$ at $633 \mathrm{~nm}, V=5.67$ at $830 \mathrm{~nm}$ ), the simplified formula drastically overestimated the loss, with greater error for larger fiber $V$ number. Stress was accounted for in both simulation and formula, which was necessary for the good agreement between simulation and experiment.

about the mode distributions themselves, other than that they be localized near the fiber core. We may therefore proceed with some confidence in the ability of the BPM to predict bend loss, not only for the fundamental mode, but also those of higher order. In the next section, this ability is utilized to gauge the accuracy of different analytical bend loss formulae. As will be seen, excellent agreement between theory and simulation, for a variety of fiber modes, provides further support for this claim.

\section{COMPARISON With ANALYTICAL FORMUlaS}

In order to examine the discrepancy between the simplified bend loss formula and the simulated and experimental results for multimode fiber, it is useful to consider the derivation of (9), [1], [19]. A number of simplifying assumptions were made to arrive at this formula, most notably that the modes must not change appreciably due to bending. The modal propagation constants, which vary with position in the bent fiber as shown in Fig. 2(a), were also assumed to match those of the straight fiber at $x=0$.

If instead the mode field distributions in the bent fiber are not assumed, and the fact that the propagation constant varies with position

$$
\beta_{z}(x)=\frac{\mu}{(R+x)_{\mathrm{eff}}}
$$

is considered, then a more broadly applicable formula for bend loss may be derived. In (12) $\mu$ is the angular propagation constant of the mode as it rotates about the center of curvature, and $(R+x)_{\text {eff }}$ is defined in analogy to $R_{\text {eff }}$. Following a derivation based on [1] and [19], it may be shown that a relatively general formula for bend loss is given by

$$
2 \alpha=\frac{\int_{-\infty}^{\infty}\left|\frac{\Im_{y}\left(U_{x=a}\right)}{H_{\mu}^{(2)}\left[\beta_{x}(R+a)_{\text {eff }}\right]}\right|^{2} d \beta_{y}}{\pi^{2} k_{\text {clad }}(R+a)_{\text {eff }}} .
$$


Here $\Im_{y}$ is the Fourier transform

$$
\Im_{y}\left(U_{x=a}\right)=\int_{-\infty}^{\infty} U_{x=a} \exp \left(i y \beta_{y}\right) d y
$$

$\beta_{y}$ is the angular spatial frequency in the $y$-direction, $U(x, y)$ is the normalized mode field distribution of the bent fiber

$$
U=\frac{E \sqrt{n}}{\sqrt{\iint n|E|^{2} d x d y}}
$$

and $E$ is the electric field. The numerator in the integral of (13) is therefore the spatial Fourier power spectrum of the mode, along the boundary surface $x=a$. The denominator in the integral of (13) is a Hankel function of the second kind, where

$$
\beta_{x}=\sqrt{\beta_{y}^{2}+\beta_{z(x=a)}^{2}-k_{\text {clad }}^{2}}
$$

The only major assumptions leading to (13) are those of weak guidance $\left(\mathrm{NA}_{\text {index }}^{2} \ll 2 n_{\text {core }}^{2}\right)$ and slow bending $(a \ll R)$.

In the derivation of (9), the Hankel function of (13) was originally approximated as [19]

$$
\begin{aligned}
& H_{\mu}^{(2)}\left[\beta_{x}(R+a)_{\mathrm{eff}}\right] \\
& \quad \approx i \sqrt{\frac{2}{\pi \gamma R_{\mathrm{eff}}}} \exp \left[\left(\frac{\gamma^{3}}{3 \beta_{z}^{2}}+\frac{\gamma \beta_{y}^{2}}{2 k_{\text {clad }}^{2}}\right) R_{\mathrm{eff}}-\gamma a_{\mathrm{eff}}\right] .
\end{aligned}
$$

This approximation was reached by expanding the argument of the Hankel function about $x=0$ and dropping higher order terms. The propagation constant and $\gamma$ were also evaluated at $x=0$ in (17). However, the fields and propagation constant most relevant to the bend loss in (13) are those at $x=a$ rather than the origin. It would therefore seem more appropriate to expand the argument of the Hankel function about $x=a$, which leads to the modified approximation

$$
\begin{aligned}
& H_{\mu}^{(2)}\left[\beta_{x}(R+a)_{\mathrm{eff}}\right] \\
& \quad \approx i \sqrt{\frac{2}{\pi \gamma(R+a)_{\mathrm{eff}}}} \exp \left[\left(\frac{\gamma^{3}}{3 \beta_{z}^{2}}+\frac{\gamma \beta_{y}^{2}}{2 k_{\mathrm{clad}}^{2}}\right)(R+a)_{\mathrm{eff}}\right]
\end{aligned}
$$

where $\beta_{z}$ and $\gamma$ are evaluated instead at $x=a$.

For small values of $\gamma a$, this modification to the Hankel function approximation has relatively little impact on the computed bend loss. However, for larger core sizes, the difference amounts of many orders of magnitude. To test the accuracy of the different forms, the calculated modes from the BPM simulations, and their simulated propagation constants, were used to numerically evaluate (13). The results using the modified expansion of the Hankel function in (18) are presented in Figs. 9 and 10. As can be seen, the BPM simulations agreed extremely well with the loss formula in (13), given the modified Hankel function expansion. The relative error between simulation and formula averaged less than $1 \%$, for all data points, with an average deviation of $10 \%$. This was extremely good agreement considering a

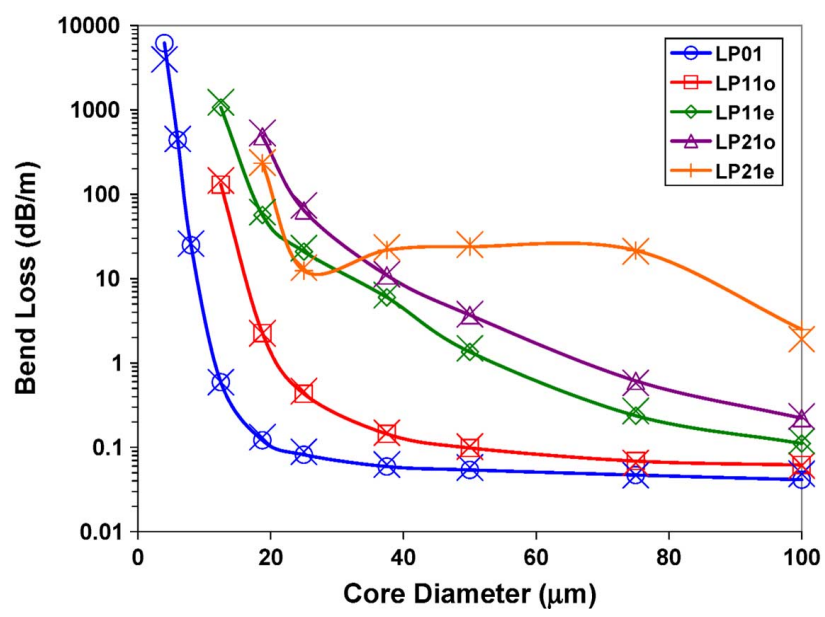

Fig. 9. Comparison of theoretical bend loss data for the lowest order fiber modes. BPM simulated loss is marked by symbols as indicated in the legend, connected by solid lines. Loss calculated directly from the simulated mode field distributions, using (13) and the modified Hankel function approximation (18), is indicated by $\times$ markers. Loss calculated directly from the mode field distributions was in excellent agreement with that obtained by propagation simulations with the BPM. If the usual Hankel function approximation in (17) was instead used, it overestimated the loss by many orders of magnitude, by the factor $\exp (2 \gamma a) \sim \exp (2 V)$. The loss of each mode was seen to stabilize with increasing core diameter, contrary to predictions made by the simplified loss formula. Loss of the different modes also converged with increasing core diameter. Simulation parameters were $\lambda_{o}=1064 \mathrm{~nm}, \mathrm{NA}_{\text {index }}=0.1$, and $R_{\text {eff }}=1.24 \mathrm{~cm}$

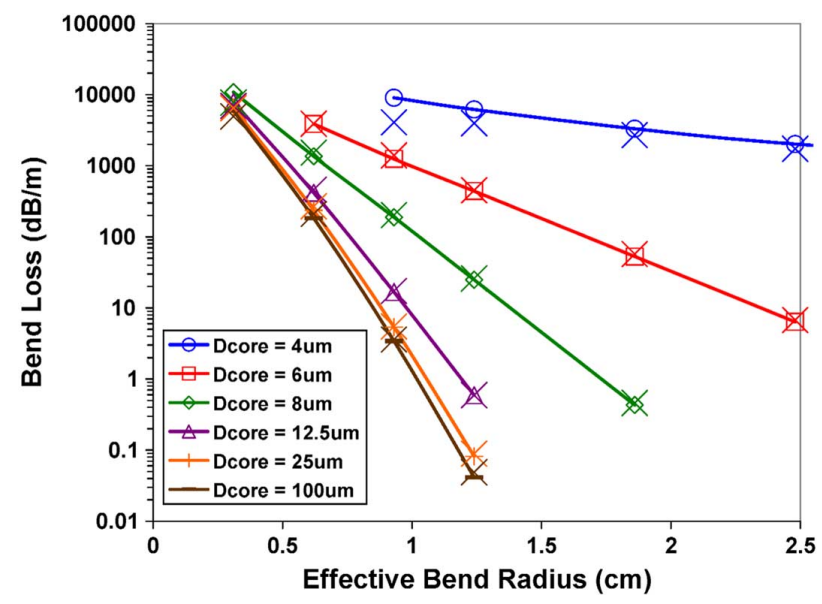

Fig. 10. Comparison of theoretical bend loss data for the fundamental fiber mode. BPM simulated loss is marked by symbols as indicated in the legend, connected by solid lines. Loss calculated directly from the simulated mode field distributions, using (13) and the modified Hankel function approximation (18), is indicated by $\times$ markers. Loss calculated directly from the mode field distributions was in excellent agreement with that obtained by propagation simulations with the BPM. If the usual Hankel function approximation in (17) was instead used, it overestimated the loss by the factor $\exp (2 \gamma a) \sim \exp (2 \mathrm{~V})$, many orders of magnitude. Simulation parameters were $\lambda_{o}=1064 \mathrm{~nm}$ and $\mathrm{NA}_{\text {index }}=0.1$.

variation of over five orders of magnitude in the data. By comparison, when the Hankel function expansion of (17) was instead used, the predicted loss was overestimated by the factor $\exp (2 \gamma a) \sim \exp (2 V)$. For the largest simulated core diameter, $100 \mu \mathrm{m}$, this amounted to an error of 25 orders of magnitude. In light of this, the modified Hankel function approximation in (18) was the clear favorite. 


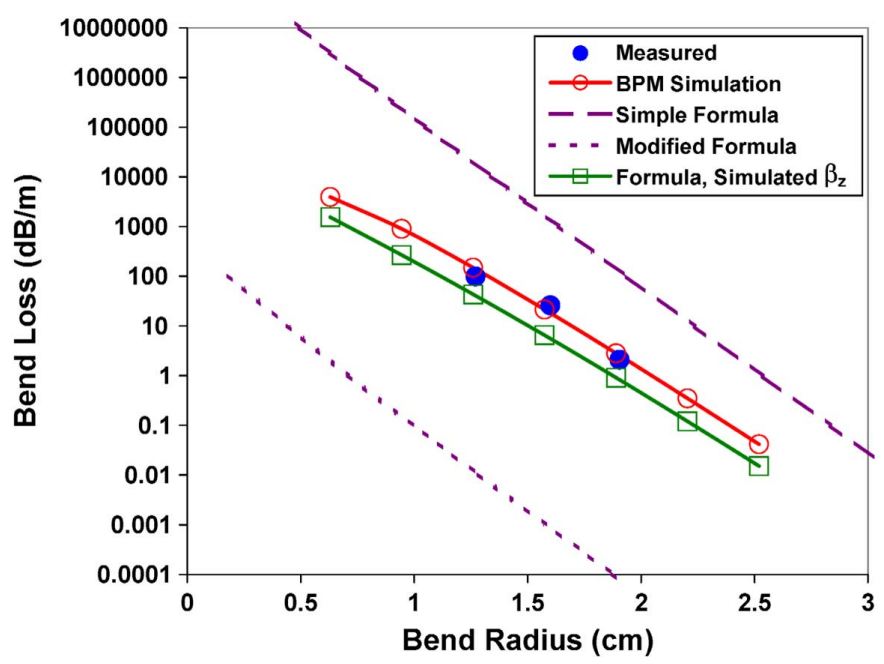

Fig. 11. Comparison of different theoretical predictions and experimental data for the fundamental mode bend loss of Liekki passive 25/240DC fiber at $633 \mathrm{~nm}$. BPM simulated loss $(\bigcirc)$ is in good agreement with measured data $(\bullet)$. The simplified loss formula, (9), overestimated the loss by orders of magnitude. Using the modified Hankel function approximation, (20) underestimated the loss by orders of magnitude. However, when the propagation constant of the bent fiber, rather than the straight fiber, was used in the modified formula $(\square)$, the agreement was much better, within a factor of about 2.5. The remaining factor of 2.5 was due to the modified, simplified formula not accounting for mode field distortion due to the bend.

When the modified Hankel function approximation (18) is inserted into (13), the loss formula becomes

$$
\begin{aligned}
2 \alpha= & \left(\frac{\gamma}{2 \pi k_{\text {clad }}}\right) \exp \left[\frac{-2 \gamma^{3}(R+a)_{\mathrm{eff}}}{3 \beta_{z}^{2}}\right] \\
& \times \int_{-\infty}^{\infty}\left|\Im_{y}\left(U_{x=a}\right)\right|^{2} \exp \left[\frac{-\gamma \beta_{y}^{2}(R+a)_{\mathrm{eff}}}{k_{\mathrm{clad}}^{2}}\right] d \beta_{y} .
\end{aligned}
$$

Agreement between simulation and this formula was excellent. The only discrepancy occurred for weakly confined, tightly bent fiber ( $2 \mathrm{a}=4 \mu \mathrm{m}, \mathrm{V}=1.18)$, as shown in Fig. 10. This was attributed to error in the simulated mode distribution, due to the truncated index profile.

\section{ERROR IN THE SIMPLIFIED FORMULA}

If we were to use the modified Hankel function expansion in (18) to re-derive the simplified bend loss formula, the following modified formula would result

$$
2 \alpha=\frac{\pi^{1 / 2} \kappa^{2} \exp \left[-\frac{2 \gamma^{3}(R+a)_{\mathrm{eff}}}{3 \beta_{z}^{2}}-2 \gamma a\right]}{2(R+a)_{\mathrm{eff}}^{1 / 2} \gamma^{3 / 2} V^{2} K_{m-1}(\gamma a) K_{m+1}(\gamma a)} .
$$

The bend loss predicted by this modified formula for the Liekki multimode fiber is shown in Fig. 11. As can be seen, although the original formula overestimated the bend loss, using the modified Hankel function approximation caused the simplified formula to underestimate it by a similar amount.

The fact that the modified Hankel function approximation did not provide better results when used in the simplified formula, while it did in the previous section, provides insight into the limitations of the simplified formula itself. This formula accounts for neither the mode field deformation, nor the weaker mode confinement (as expressed by the reduced propagation constant at $x=a$ ), that occurs within the bent fiber [1]. We can gauge the relative importance of these two simplifications, however, by inserting the correct propagation constants from BPM simulations into (20). Fig. 11 illustrates the results. By using the correct propagation constant in (20), the relative error in the loss predictions was reduced from a few orders of magnitude to a factor of about 2.5. The remaining error was attributable to not accounting for the mode field deformation. Clearly, not adjusting the propagation constant was the dominant source of error in this case.

This result helps explain why the simplified bend loss formula in (9) works relatively well for single-mode fiber, but not multimode. Although (20) includes the preferred Hankel function approximation, it tends to underestimate the loss by not accounting for the change in propagation constant. Equation (9), on the other hand, is greater than (20) by the factor $\exp (2 \gamma a)$. For single-mode fiber this factor is sufficient to make up the difference, providing a reasonably good estimate of the bend loss. For multimode fiber, however, this factor grows much too large to provide a good assessment.

\section{DISCUSSION}

Before concluding it is worth commenting on the physical interpretation of these results. Equation (13) states simply that fiber bend loss may be determined by expanding the fields on the boundary cylinder, defined by $x=a$, as a superposition of outgoing cylindrical waves in the cladding. The amount of loss depends upon the strength of the mode fields on this surface, and which cylindrical waves (Hankel functions) the fields excite. The latter are determined by the propagation constant at the boundary surface, as well as the field variation in the y-direction. Note also that there is no requirement that the waveguide be a simple step-index fiber in this formula. Equation (19) is therefore generally applicable to waveguides that have both a uniform dielectric, and modal caustic boundaries, located in the region $x>a$.

It is also worth comparing the results of this paper to conclusions drawn previously about field deformation in bent fibers. A previous interpretation was that field deformation decreases the fundamental mode bend loss in multimode fibers [3]. This is despite the fact that the fields became stronger on the boundary surface, and was based on a comparison between numerical predictions and (9). The alternate interpretation offered here would seem more intuitive: that the modified simplified formula of (20) underestimates the loss, but the corrections of field deformation toward the outer edge of the fiber and weaker confinement (reduced propagation constant at $x=a$ ) cause it to increase. The tendency for modal loss to stabilize with increasing core size in Figs. 9 and 10 is also in stark contrast to previous predictions based on the simplified formula. This is due to the fiber modes transitioning to whispering gallery modes at sufficiently large core diameter. 
The loss of the odd LP modes is also quite notable, given that bending causes them to decouple from the even LP modes in the bent fiber. If one uses the simplified loss formula, their loss is predicted to be negligible. This is not significant if each of these modes is coupled to one of the lossy, even LP modes, because the total loss is then averaged over the even and odd constituents [1]. However, when uncoupled, the odd LP modes can have significantly lower loss, which can be quite important. This is apparent in Fig. 9, which shows that the losses of the $\mathrm{LP}_{01}$ and $\mathrm{LP}_{11 \mathrm{o}}$ modes become similar at larger core sizes. This has serious implications for the effectiveness of mode stripping using bend loss in multimode fiber amplifiers. For example, at a core diameter of $25 \mu \mathrm{m}$, the differential bend loss between the fundamental and $\mathrm{LP}_{11 o}$ modes was simulated to be $0.35 \mathrm{~dB} / \mathrm{m}$. The simplified formula, in contrast, predicts differential loss on the order of $5 \mathrm{~dB} / \mathrm{m}[4]$.

Lastly, it is important to note that by assuming an infinite cladding, (19) does not account for possible back reflections from the fiber jacket, or from additional cladding layers. Thus, although this loss formula predicts the power flow away from the fiber core, it cannot predict the fraction of the power that may eventually return. However, (19) may be readily extended to fibers with high-index jackets $\left(n_{\text {jacket }}>n_{\text {clad }}\right)$ using the approach detailed in [20]. This results in an oscillatory loss spectrum, with maxima and minima given by

$$
2 \alpha_{\text {coated }}= \begin{cases}2 \alpha \eta^{1 / 2}, & \max \\ 2 \alpha \eta^{-1 / 2}, & \min \end{cases}
$$

where $2 \alpha$ is determined from (19), and

$$
\eta=\left(\frac{n_{\text {clad }}}{n_{\text {jacket }}}\right)^{4 / 3}\left[\frac{2 \Delta a k_{\text {jacket }}^{2}-R_{\text {eff }} \beta_{x p}^{2}}{2 \Delta a k_{\text {clad }}^{2}-R_{\text {eff }} \beta_{x p}^{2}}\right] .
$$

Here $\Delta a$ is the difference between the core and cladding radii $\left(a_{\text {clad }}-a_{\text {core }}\right)$, and $\beta_{x p}$ is similar to (16) but with $\left|\beta_{y}\right|$ replaced by the peak spatial frequency from the modal Fourier expansion (14), which is zero for even LP modes, but nonzero for odd. The extremities in the bend loss correspond to the conditions

$$
\frac{4 a_{\mathrm{clad}} \beta_{x p}}{3 \pi}\left(\frac{R_{\mathrm{eff}}}{R_{c}}\right)\left(\frac{R_{c}}{R_{\mathrm{eff}}}-1\right)^{3 / 2}= \begin{cases}2 m-1 / 2, & \max \\ 2 m-3 / 2, & \min \end{cases}
$$

where

$$
R_{c}=\frac{2 a_{\text {clad }} k_{\text {clad }}^{2}}{\beta_{x p}^{2}}
$$

Furthermore, these oscillations disappear for large bend radii, $R>R_{c}$, such that the caustic boundary lies outside of the fiber cladding.

For low-index fiber jackets $\left(n_{\text {jacket }}^{2}<n_{\text {clad }}^{2}-\mathrm{NA}_{\text {index }}^{2}\right)$ on the other hand, leakage from cladding to jacket is relatively weak, so light radiated from the core can accumulate substantially in the cladding. In such cases, (19) merely describes the rate of outward power flow from the fiber core. This must be balanced against the inward flow due to reflections from the cladding-jacket interface, which is rather complicated, and beyond the scope of this paper. Equation (19) is therefore quite applicable, but should be used with caution when applied to low-index jacketed or doubly clad fibers.

\section{CONCLUSION}

Although the BPM simulations presented in this paper, and the analytical formula of (19), represent vastly different theoretical approaches to predicting bend loss, they agreed remarkably well for both single-mode and multimode fibers, and fundamental and higher order modes. This strongly suggests the soundness of both approaches. Their agreement with experiment also indicates a good degree of accuracy.

One consequence is that this demonstrates the ability of the beam propagation method with conformal mapping to accurately predict both the modes of a bent fiber, and their propagation characteristics. This suggests that the BPM may also be able to handle more complicated bent waveguide structures, which are currently not well understood.

A second consequence is that the loss formula introduced in (19) can accurately predict waveguide bend loss, without the need for BPM simulation. This offers a simple approach, which only requires prior knowledge of the mode field distribution and the propagation constant. Various mode-solving techniques may therefore be used in conjunction with (19) to predict bend loss. This approach is also broadly applicable to more complicated fiber geometries.

Lastly, the results of this paper provide revised guidelines for use of simplified bend loss formulae. For single-mode fiber, the unmodified, simplified formula works well provided that stress is accounted for through the effective bend radius. For multimode fiber, the modified formula in (20) provides reasonable results, but requires some knowledge of the propagation constant variation with bending.

\section{APPENDIX A \\ SiMULATION ACCURACY}

To estimate the impact of simulation grid size on the mode calculations, a series of simulations were performed for the fundamental mode of the straight fiber discussed in Fig. 4, using different transverse $(\Delta x, \Delta y)$ and longitudinal $(\Delta z)$ steps and a launched Gaussian distribution. The resulting normalized propagation constants $\left(b_{\text {sim }}\right)$ are compared to the exact value $\left(b_{\text {exact }}=0.9177957658\right)$ computed from the characteristic equation [19] in Fig. 12. As shown, the simulations were extremely accurate at the step sizes used in this paper $(\Delta x=\Delta y=0.1 \mu \mathrm{m}$ and $\Delta z=0.5 \mu \mathrm{m})$, as well as for somewhat larger grids.

To estimate the impact of truncating the refractive index profile on the mode calculations, a series of simulations were performed for the fundamental mode of the fiber in Fig. 4, using various refractive index transition points, $x_{\text {trans }}$, and bend radii. The resulting normalized propagation constants $\left(b_{\text {sim }}\right)$ are compared to those computed with $x_{\text {trans }}$ just inside the caustic boundary $\left(b_{\text {caustic }}\right)$ in Fig. 13 . Noting that $b_{\text {caustic }}$ should approach the exact value as the caustic boundary moves farther outward, it is evident that the curve for $R_{\text {eff }}=1.24$ must closely approximate the relative error in the calculation caused by truncating the index profile. Furthermore, since the other 


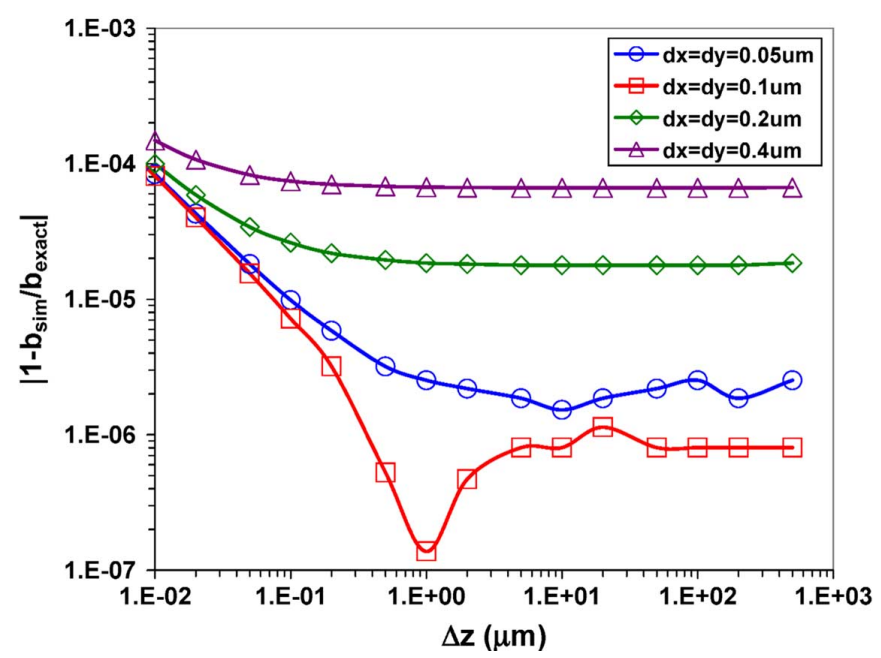

Fig. 12. Relative error in the simulated normalized propagation constant, for various simulation step sizes and the fundamental mode of the straight fiber in Fig. 4. Error was relative to the exact value calculated from the characteristic equation. Accuracy was excellent for the simulation steps used for this fiber in this paper, $\Delta x=\Delta y=0.1 \mu \mathrm{m}$, and $\Delta z=0.5 \mu \mathrm{m}$.

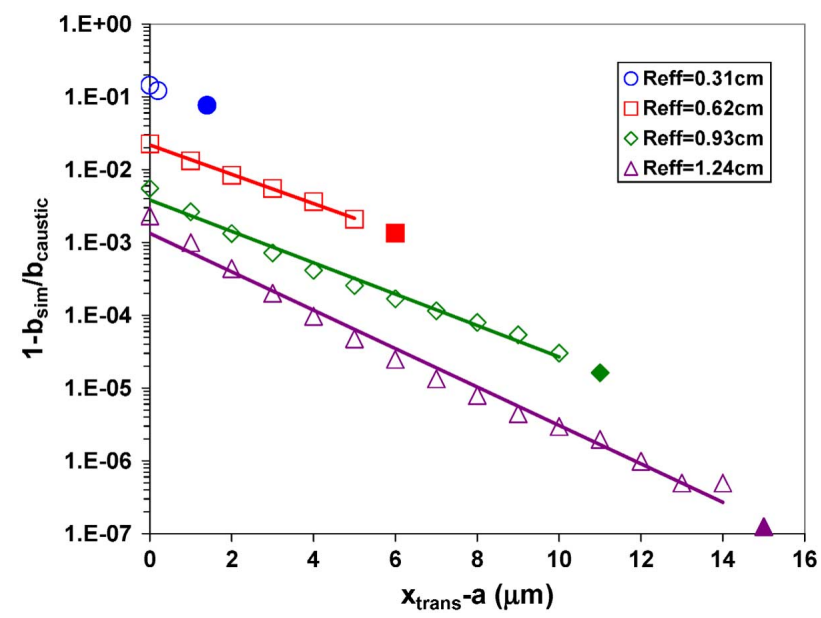

Fig. 13. Variation in simulated normalized propagation constants due to truncation of the refractive index profile in bent fibers. Open symbols denote the relative difference between $b_{\text {sim }}$ and $b_{\text {caustic }}$. Extrapolating these data sets back to $x_{\text {trans }} \approx x_{\text {caustic }}$ provides an estimate of the relative error in $b_{\text {caustic }}$, which is indicated by the filled symbols.

curves exhibit a similar exponential dependence, each may be taken as a decent estimate of the relative error. Extrapolating each curve back to the caustic then provides an estimate of the relative error in $b_{\text {caustic }}$, indicated by the filled symbols in Fig. 13.

Repeating this procedure for the various fiber modes, and also for the fundamental mode of a similar single mode fiber with V-number $2.36(2 a=4 \mu \mathrm{m})$, leads to the plot in Fig. 14. Here $b$ represented the "exact" value, corresponding to a nontruncated index profile. The plot shows a clear one-to-one relationship between the relative error in $b_{\text {caustic }}$ and the bend loss, calculated using (19). Such a result is quite intuitive, as both the loss and the error in the propagation constant depend on the fraction of the mode power at the caustic boundary. The apparent generality of this relationship also suggests an alternative method

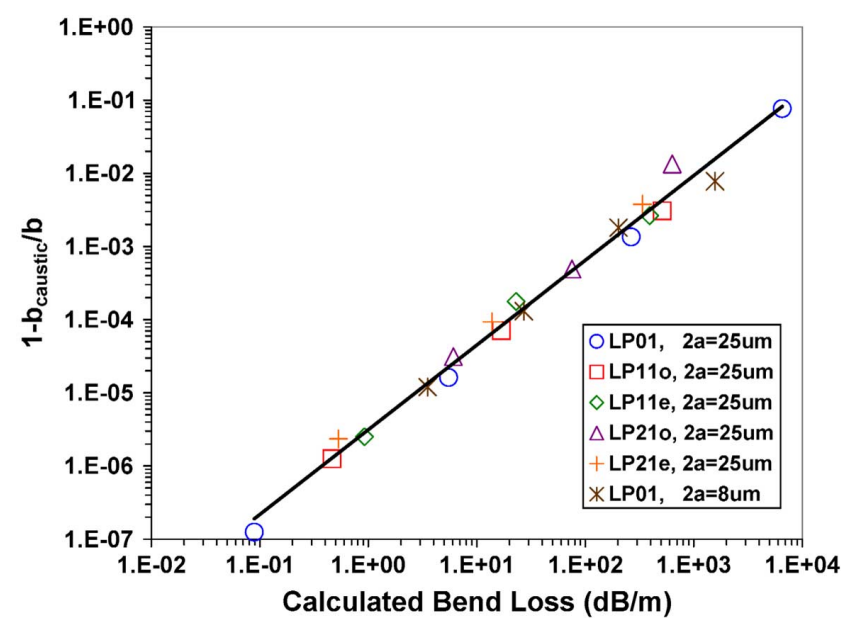

Fig. 14. Relative error in the simulated normalized propagation constant, $b_{\text {caustic }}$, caused by truncation of the refractive index profile of the bent fiber. Data shown correspond to the lowest order modes of the fiber in Fig. 4, as well as the fundamental mode of an otherwise identical fiber with core diameter 4.0 $\mu \mathrm{m}$. Loss was calculated from (19), and the trend line represents a power law fit. The common trend for the various modes and fiber parameters indicates a one-to-one relationship between bend loss and the error in the propagation constant caused by truncating the refractive index profile.

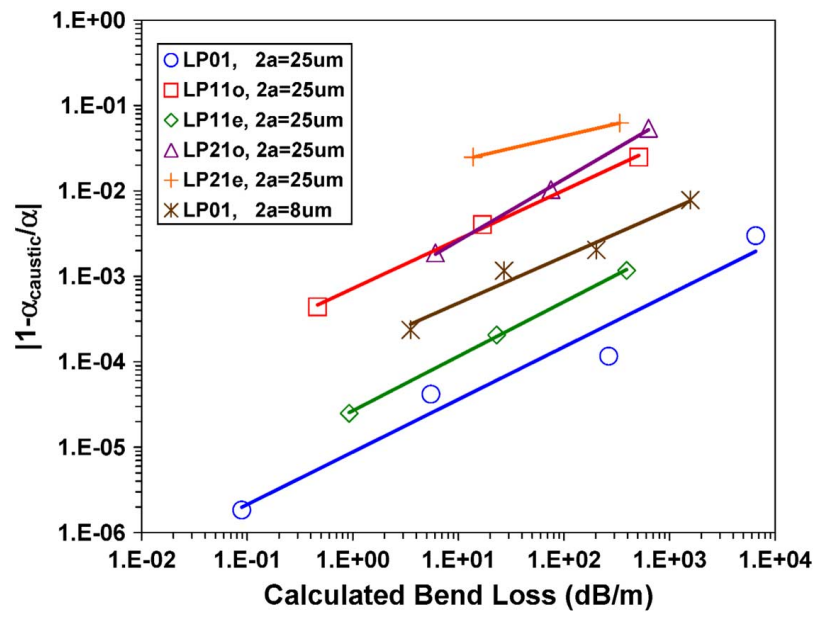

Fig. 15. Relative error in the calculated bend loss caused by truncating the refractive index profile of the bent fiber. The calculated loss refers to the formula in (19). A similar extrapolation procedure to Fig. 13 was used to determine each data point. Data shown correspond to the lowest order modes of the fiber in Fig. 4, as well as the fundamental mode of an otherwise identical fiber with core diameter $4.0 \mu \mathrm{m}$.

for predicting bend loss: using the error in the mode calculations. For the present study, however, it is sufficient to note that Fig. 14 shows that the error in the mode calculations was relatively small, even for bend loss of the order of hundreds of decibels per meter.

To estimate the impact of truncating the refractive index profile on the calculated bend loss, a similar extrapolation procedure was followed, by evaluating (19) for each of the modes simulated versus $x_{\text {trans }}$. The resulting error in the loss coefficient is displayed in Fig. 15, where $\alpha$ represents the "exact" value for a nontruncated index profile. As shown, truncating the refractive index profile led to considerable error in the loss of the highest order modes, at large values of loss. However, even in the worst 


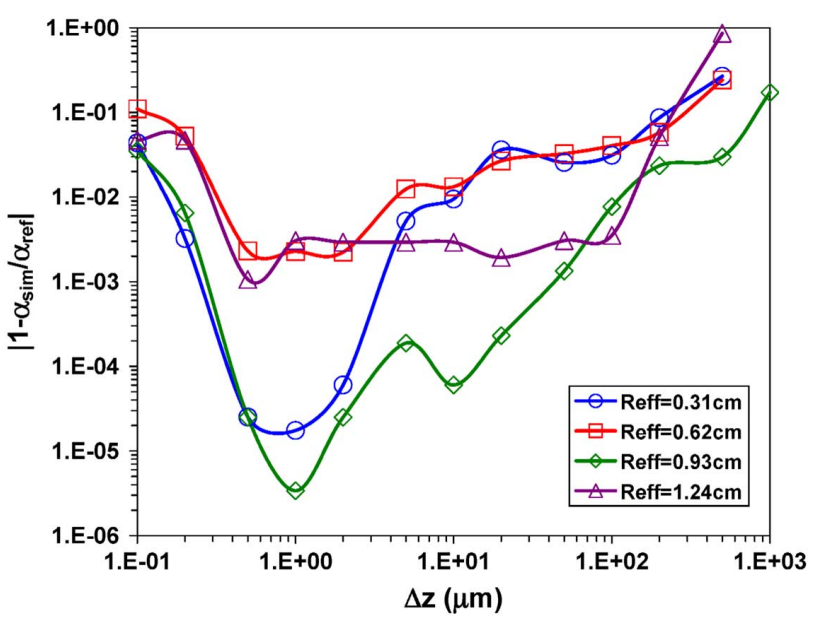

Fig. 16. Variation in the simulated bend loss for different longitudinal step sizes $\Delta \mathrm{z}$ and bend radii, for the fundamental mode of the fiber in Fig. 4. For scaling purposes, the simulated loss is compared to a reference value $\alpha_{\text {ref }}$ for each bend radius. Repeatability in the loss simulations was within $10 \%$, and in many cases much better.

case, the relative error was less than $10 \%$. For the purposes of this paper, this was quite tolerable.

To estimate the impact of grid size in the loss simulations, a series of runs were performed with different longitudinal steps and bend radii, for the fundamental mode and the fiber discussed in Fig. 4. The results are illustrated in Fig. 16, which plots the relative difference between the simulated loss and a reference value $\alpha_{\text {ref }}$ included for scaling purposes. The reference value was based on results with $\Delta z$ close to $1.0 \mu \mathrm{m}$, which were relatively consistent. As shown, the simulated loss varied by less than $10 \%$ for reasonable step sizes, which was tolerable for the purposes of this paper. To consider other modes, and other fiber parameters, it is sufficient to note that the agreement between simulated and calculated bend loss was shown to be quite good in Section IV.

\section{APPENDIX B}

\section{NECESSITY OF THE ELASTOOPTIC CORRECTION}

A great body of work demonstrates that stress plays a key role in the behavior of bent optical fibers through the elastooptic effect. Although this and many other papers indicate that elasto-optic corrections must be included in the bend loss formulae, a recent analysis has concluded otherwise [21]. This discrepancy may be explained by noting that [21] uses a significantly different value of $\mathrm{NA}_{\text {index }}$ for SMF28, 0.128, as opposed to 0.117 in this paper. The cubed ratio of these values is $(0.128 / 0.117)^{3}=1.31$, which is very close to the elasto-optic correction for the bend radius, 1.28 . Noting that the primary dependence on $\mathrm{NA}_{\text {index }}$ in (19) is through the term $R_{\mathrm{eff}} \gamma^{3}$, which is proportional to $R_{\text {eff }} \mathrm{NA}_{\text {index }}^{3}$, it is clear that use of the larger numerical aperture should allow the elasto-optic correction to be dropped. However, although both cases result in similar predictions of bend loss, only one can be correct.

A literature search for the numerical aperture of SMF28 does not readily solve the problem, as values range broadly between 0.12 and 0.14 . It should be cautioned, however, that quoted values are typically based on measurements of beam output,
TABLE I

SPECIFICATIONS FOR SMF28 FIBER

\begin{tabular}{||c|c|c|c|c||}
\hline \hline & $\begin{array}{c}\text { Quoted } \\
\text { Value }\end{array}$ & $\begin{array}{c}\text { Computed } \\
\mathrm{w} / N A_{\text {index }} \\
0.128\end{array}$ & $\begin{array}{c}\text { Computed } \\
\mathrm{w} / N A_{\text {index }} \\
0.123\end{array}$ & $\begin{array}{c}\text { Computed } \\
\mathrm{w} / N A_{\text {index }} \\
0.117\end{array}$ \\
\hline $\begin{array}{c}\text { Cutoff Wavelength } \\
(\mathrm{nm})\end{array}$ & $<1260$ & 1370 & 1320 & 1250 \\
\hline $\begin{array}{c}M F D(\mu \mathrm{m}) \\
\text { at } 1310 \mathrm{~nm}\end{array}$ & $9.2 \pm 0.4$ & 8.75 & 8.98 & 9.29 \\
\hline $\begin{array}{c}M F D(\mu \mathrm{m}) \\
\text { at } 1550 \mathrm{~nm}\end{array}$ & $10.4 \pm 0.8$ & 9.86 & 10.20 & 10.66 \\
\hline
\end{tabular}

which can be entirely inconsistent with the index-based numerical aperture given by (6). For example, manufacturer specifications for SMF28 fiber [22] list the numerical aperture as 0.14, but also state the index step as $0.36 \%$, which leads to $\mathrm{NA}_{\text {index }}$ of 0.123 . To complicate things further, the index step is usually measured in the pre-form, prior to drawing the fiber, rather than in the fiber itself. This may be the reason the cutoff wavelength

$$
\lambda_{\mathrm{oc}}=\frac{2 \pi a \mathrm{NA}_{\text {index }}}{2.405}
$$

computed using $\mathrm{NA}_{\text {index }}$ of 0.123 and $1320 \mathrm{~nm}$ is significantly larger than the manufacturer's specification of $<1260 \mathrm{~nm}$. Such a value would imply multimode behavior into the $1310-\mathrm{nm}$ operations band, which is not the case.

A more sensitive indicator of $\mathrm{NA}_{\text {index }}$ in drawn fiber is the cutoff wavelength, given by (B1). The 1260-nm upper bound quoted for the cutoff wavelength implies that $\mathrm{NA}_{\text {index }}$ should remain less than 0.118 . This is the primary reason behind choosing 0.117 in this paper. Table I also shows that this value provided good agreement with quoted mode field diameters (MFD) at both 1310 and $1550 \mathrm{~nm}$, as calculated using [23]

$$
\mathrm{MFD} \approx 2 a\left(0.65+1.619 \mathrm{~V}^{-3 / 2}+2.879 \mathrm{~V}^{-6}\right) .
$$

In addition, Table I compares listed specifications to those calculated using a numerical aperture of 0.128 . As shown, agreement was relatively poor. This, along with the existing body of work, leads to the conclusions that the correction for elasto-optic effects in bend loss calculations is indeed necessary, contrary to [21].

\section{ACKNOWLEDGMENT}

The authors would like to thank C. A. Villarruel, A. M. Kowalevicz, G. A. Miller, and R. P. Moeller for illuminating discussions on this subject.

\section{REFERENCES}

[1] D. Marcuse, "Curvature loss formula for optical fibers," J. Opt. Soc. Amer. B, vol. 66, pp. 216-220, Mar. 1976.

[2] A. B. Sharma, A. H. Ai-Ani, and S. J. Halme, "Constant-curvature loss in monomode fibers: An experimental investigation," Appl. Opt., vol. 23, pp. 3297-3301, Oct. 1984.

[3] D. Marcuse, "Field deformation and loss caused by curvature of optical fibers," J. Opt. Soc. Amer., vol. 66, no. 1, pp. 311-320, Apr. 1976.

[4] J. P. Koplow, D. A. V. Kliner, and L. Goldberg, "Single-mode operation of a coiled multimode fiber amplifier," Opt. Lett., vol. 25, pp. 442-444, Apr. 1, 2000.

[5] R. Scarmozzino et al., "Numerical techniques for modeling guidedwave photonic devices," IEEE J. Sel. Topics Quantum Electron., vol. 6, pp. 150-162, Jan.-Feb. 2000. 
[6] M. Heiblum and J. H. Harris, "Analysis of curved optical waveguides by conformal transformation," IEEE J. Quantum Electron., vol. QE-11, no. 2, pp. 75-83, Feb. 1975.

[7] J. Yamauchi et al., "Beam propagation analysis of bent step-index slab waveguides," Electron. Lett., vol. 26, pp. 822-824, June 7, 1990.

[8] J. Yamauchi et al., "Analysis of bent asymmetric slab waveguides by the beam propagation method," Opt. Commun., vol. 79, pp. 291-294, Nov. 1, 1990

[9] D. Marcuse, "Influence of curvature on the losses of doubly clad fibers," Appl. Opt., vol. 21, pp. 4208-4213, Dec. 1, 1982.

[10] R. Ulrich, S. C. Rashleigh, and W. Eickhoff, "Bending-induced birefringence in single-mode fibers," Opt. Lett., vol. 5, pp. 273-275, June 1980.

[11] A. D. Kersey et al., "Fiber grating sensors," J. Lightw. Technol., vol. 15, no. 8, pp. 1442-1463, Aug. 1997.

[12] K. Nagano, S. Kawakami, and S. Nishida, "Change of the refractive index in an optical fiber due to external forces," Appl. Opt., vol. 17, pp. 2080-2085, July 1, 1978.

[13] W. P. Huang and C. L. Xu, "Simulation of three-dimensional optical waveguides by a full-vector beam propagation method," IEEE $J$. Quantum Electron., vol. 29, no. 10, pp. 2639-2649, Oct. 1993.

[14] G. R. Hadley, "Transparent boundary condition for the beam propagation method," IEEE J. Quantum Electron., vol. 28, no. 1, pp. 363-370, Jan. 1992.

[15] A. W. Snyder and W. R. Young, "Modes of optical waveguides," $J$. Opt. Soc. Am., vol. 68, pp. 297-309, Mar. 1978.

[16] S. J. Garth, "Mode behaviour on bent planar dielectric waveguides," Proc. IEE Optoelectron., vol. 142, pp. 115-119, Apr. 1995.

[17] J. Sakai and T. Kimura, "Analytical bending loss formula of optical fibers with field deformation," Radio Sci., vol. 17, pp. 21-29, Jan.-Feb. 1982.

[18] U.S. Naval Research Lab., private communication.

[19] D. Marcuse, Light Transmission Optics, 2nd ed. New York: Van Nostrand Reinhold, 1982, pp. 406-414.

[20] H. Renner, "Bending losses of coated single-mode fibers: A simple approach," J. Lightw. Technol., vol. 10, no. 5, pp. 544-550, May 1992.

[21] Q. Wang et al., "Theoretical and experimental investigations of macrobend losses for standard single mode fibers," Opt. Expr., vol. 13, pp. 4476-4484, Jun. 2005.

[22] "Corning SMF28 Optical Fiber," Corning, Inc., Corning, NY, Apr. 2002.

[23] D. Marcuse, "Gaussian approximation of the fundamental modes of graded-index fibers," J. Opt. Soc. Amer., vol. 68, pp. 103-109, Jan. 1978.

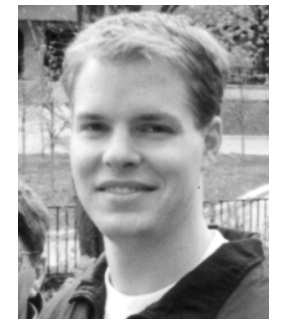

Ross T. Schermer (M'01) was born in Rock Island, IL, in 1977. He received the B.A. degree in physics from Gustavus Adolphus College, St. Peter, MN, in 1999, and the M.S. and Ph.D. degrees in electrical engineering from the University of Minnesota, Minneapolis, in 2002 and 2004, respectively. His doctoral research was on erbium-doped optical amplifiers in amorphous zirconia, and directional coupler modulators with linear response.

Since 2004, he has held a Postdoctoral Research Associateship at the U.S. Naval Research Laboratory, Washington, DC, supported by a grant from the National Research Council. His research interests include optical devices for photonic communications and sensing, optoelectronic materials and theory and design of guided-wave devices. His current research deals with high-performance modulators and switches, large mode area fiber amplifiers, and optical sensors.

Dr. Schermer is a member of the Optical Society of America, and an officer of the Optical Society of America's National Capital Section.

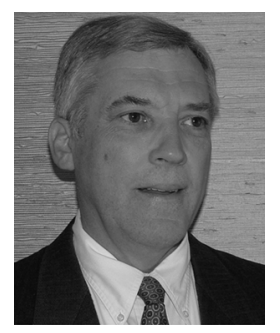

James H. Cole was born in New London, CT, in 1948. He received the B.S. degree from Bucknell University, Lewisburg, PA, in 1970, and the M.S. degree from Pennsylvania State University, State College, in 1972, both in physics.

In 1973, he joined the TRW Space Systems. He moved to the Naval Research Laboratory (NRL), Washington, DC, in 1978, continuing his work in fiber optic acoustic sensors, nonacoustic fiber sensors, and interferometric demodulation. In 1986, he left NRL to form Dylor Corporation where he worked in microwave transmission over fibers as well as continuing fiber sensor research. In 2000, he returned to NRL, where he holds the position of Research Physicist. His work includes research in both fiber and planar integrated optics devices and sensors. His work has produced several important contributions in the field of fiber-optic devices. He holds eight patents with one pending and has contributed over 80 publications and presentations.

Mr. Cole is a member of the Optical Society of America. He has received two Alan Berman Publication awards, and several Department of the Navy Invention awards. He is a Past President and Webmaster of the National Capital Section of the Optical Society of America and is Secretary of the Washington Academy of Sciences. 